\title{
Strain-induced magnetic anisotropy in epitaxial thin films of the spinel $\mathrm{CoCr}_{2} \mathrm{O}_{4}$
}

\author{
J. A. Heuver, ${ }^{1,}{ }^{*}$ A. Scaramucci, ${ }^{2,3, \dagger}$ Y. Blickenstorfer, ${ }^{2}$ S. Matzen,,${ }^{1,4}$ N. A. Spaldin, ${ }^{2}$ C. Ederer, ${ }^{2}$ and B. Noheda ${ }^{1}$ \\ ${ }^{1}$ Zernike Institute for Advanced Materials, University of Groningen, Groningen, The Netherlands \\ ${ }^{2}$ Materials Theory, ETH Zürich, Zürich, Switzerland \\ ${ }^{3}$ Laboratory for Scientific Development and Novel Materials, Paul Scherrer Institut, 5235 Villigen PSI, Switzerland \\ ${ }^{4}$ Institut d'Electronique Fondamentale, Unité Mixte de Recherche 8622, Centre National de la Recherche Scientifique, \\ and Universite Paris-Sud, 91405 Orsay, France
}

(Received 27 July 2015; revised manuscript received 17 September 2015; published 21 December 2015)

\begin{abstract}
We show that the magnetic anisotropy in spinel-structure $\mathrm{CoCr}_{2} \mathrm{O}_{4}$ thin films exhibits a strain dependence in which compressive strain induces an out-of-plane magnetic easy axis and tensile strain induces an in-plane easy axis, exactly opposite to the behavior reported for the related compound $\mathrm{CoFe}_{2} \mathrm{O}_{4}$. We use density functional theory calculations within the LSDA $+U$ approximation to reproduce and explain the observed behavior. Using second-order perturbation theory, we analyze the anisotropy tensor of the $\mathrm{Co}^{2+}$ ions in both octahedral and tetrahedral coordination, allowing us to extend our results to spinels with general arrangements of $\mathrm{Co}^{2+}$ ions.
\end{abstract}

DOI: 10.1103/PhysRevB.92.214429

PACS number(s): 75.70.Ak, 75.80.+q, 75.30.Gw, 71.15.Mb

\section{INTRODUCTION}

Thin films with out-of-plane spontaneous magnetization, showing so-called perpendicular magnetic anisotropy, are of great interest for applications such as high-density magnetic memories with fast switching [1]. Perpendicular magnetic anisotropy is also needed for spintronic applications, for example, in magnetic tunnel junctions, and for low-energy current-driven domain-wall motion [2-5].

In a system of finite size, magnetic anisotropy is determined by the balance of magnetocrystalline and shape anisotropy. The former is a bulk property originating from spin-orbit interaction, while the latter originates from magnetic dipoledipole interaction and depends on the geometry of the sample. For the case of thin films, where shape anisotropy always tends to favor in-plane magnetization [6], it is interesting to understand how the strain affects the magnetocrystalline part. Indeed, the way in which epitaxial strain can affect the magnetocrystalline anisotropy has been the subject of intensive investigations [7-14]. A prominent example of such investigations is the spinel compound $\mathrm{CoFe}_{2} \mathrm{O}_{4}$, which has a strong magnetostriction coefficient $[8,11,12,15]$. In $\mathrm{CoFe}_{2} \mathrm{O}_{4}$ thin films, it was shown that changing the sign of the strain leads from cooperation to competition of shape and magnetocrystalline anisotropies [8].

Compounds with spinel $\left(\mathrm{MgAl}_{2} \mathrm{O}_{4}\right.$-type $)$ structure have chemical formula $A B_{2} X_{4}$ where $A$ and $B$ are cations and $X$ represents the ligand anion (usually $\mathrm{O}, \mathrm{S}$, or $\mathrm{Se}$ ). In this structure, the cations occupy either the tetrahedrally coordinated [16] (T) site or the octahedrally coordinated $(\mathbf{O})$ site. There are twice as many $\mathbf{O}$ sites as $\mathbf{T}$ sites. In a normal spinel, $A$ and $B$ cations occupy $\mathbf{T}$ and $\mathbf{O}$ sites, respectively, while in an inverse spinel half of the $B$ cations occupy the $\mathbf{T}$ sites and the remaining half occupy the $\mathbf{O}$ sites together with the $A$ cations. In general, spinels can be characterized by the degree of inversion (i.e., the concentration of $B$ cations occupying $\mathbf{T}$ sites).

\footnotetext{
*j.a.heuver@rug.nl

†andrea.scaramucci@psi.ch
}

The bulk structure (unstrained case) of the inverse spinel $\mathrm{CoFe}_{2} \mathrm{O}_{4}$, with a disordered $\mathrm{Co}^{2+} / \mathrm{Fe}^{3+}$ occupancy on the octahedrally coordinated sites, has cubic symmetry (space group $F d \overline{3} m$ ). This forces the quadratic magnetization terms in the magnetocrystalline anisotropy to vanish, leaving as lowest-order terms the quartic ones, which are typically of smaller size, although they can be appreciably large $[12,17]$. However, strain lifts the cubic symmetry, allowing quadratic magnetocrystalline anisotropy, which can either favor in-plane or out-of-plane magnetization. While in $\mathrm{CoFe}_{2} \mathrm{O}_{4}$ compressive strain favors in-plane magnetization, i.e., magnetocrystalline and shape anisotropies cooperate $[8,11,13,18]$, in $\mathrm{CoFe}_{2} \mathrm{O}_{4}$ films grown under tension on $\mathrm{MgO}$, the strain-induced out-ofplane bulk anisotropy is large enough to overcome the shape anisotropy, promoting out-of-plane magnetization [7-10,14]. The magnetocrystalline anisotropy in $\mathrm{CoFe}_{2} \mathrm{O}_{4}$ is expected to be the result of strong spin-orbit coupling at the $\mathrm{Co}^{2+}$ cation $[19,20]$ and, thus, a significant effect is expected for different site occupation of the $\mathrm{Co}^{2+}$ ions [21,22], as observed in $\mathrm{CoFe}_{2} \mathrm{O}_{4}$ under different annealing conditions [11].

Another $\mathrm{Co}^{2+}$ compound with cubic spinel structure, $\mathrm{CoCr}_{2} \mathrm{O}_{4}$, raised considerable interest a few years ago as it was discovered to be a multiferroic with strong magnetoelectric coupling when undergoing a transition to a ferrimagnetic conical spiral state [23-26]. As temperature is lowered, $\mathrm{CoCr}_{2} \mathrm{O}_{4}$ first shows a transition to a collinear ferrimagnetic state at $\mathrm{T}_{N 1} \sim 95 \mathrm{~K}$, which exists down to $\mathrm{T}_{N 2} \sim 26 \mathrm{~K}$. Below this temperature the ferrimagnetic conical spiral state appears and induces an electric polarization [23,26]. At $\mathrm{T} \sim 14 \mathrm{~K}$ an abrupt change in electric polarization occurs $[23,26]$. The fact that it is difficult to grow good quality single crystals leaves some open questions on the detailed magnetic state [26,27]. To date, the epitaxial growth of $\mathrm{CoCr}_{2} \mathrm{O}_{4}$ in thin-film form has only been reported by a few groups [28-31]. No study so far has reported strain engineering of magnetic properties.

$\mathrm{CoCr}_{2} \mathrm{O}_{4}$ has a strong tendency to order in a normal spinel structure [32], i.e., with $\mathrm{Co}^{2+}$ occupying $\mathbf{T}$ sites and $\mathrm{Cr}^{3+}$ occupying the $\mathbf{O}$ sites. This is different from the behavior of $\mathrm{CoFe}_{2} \mathrm{O}_{4}$ and $\mathrm{NiFe}_{2} \mathrm{O}_{4}$, which have an inverse spinel structure, with the $\mathrm{Co}^{2+}$ and $\mathrm{Ni}^{2+}$ occupying $\mathbf{O}$ sites, while $\mathrm{Fe}^{3+}$ cations are distributed in equal proportion among $\mathbf{O}$ and 
T sites (although real samples can show partial occupation of $\mathrm{Co}^{2+}$ in $\mathbf{T}$ sites due to growth kinetic effects [11]). Because of the different $\mathrm{Co}^{2+}$ distribution in $\mathrm{CoCr}_{2} \mathrm{O}_{4}$ and $\mathrm{CoFe}_{2} \mathrm{O}_{4}$, the effect of strain on magnetic anisotropy is expected to be different in the two compounds. However, it is unknown how these differences will manifest themselves in actual films, since studies on the magnetic anisotropy of strained $\mathrm{CoCr}_{2} \mathrm{O}_{4}$, as well as a comprehensive theoretical treatment for spinels, are lacking.

In this work, we investigate experimentally and theoretically the strain dependence of magnetocrystalline anisotropy in $\mathrm{CoCr}_{2} \mathrm{O}_{4}$, with the purpose of generalizing the behavior of spinel systems in which $\mathrm{Co}^{2+}$ is expected to be the main source of magnetocrystalline anisotropy. After a brief discussion of experimental and computational details, we present our results of magnetic measurements performed for thin films of $\mathrm{CoCr}_{2} \mathrm{O}_{4}$ under both compressive and tensile strain. In particular, we find that in $\mathrm{CoCr}_{2} \mathrm{O}_{4}$ strain affects magnetocrystalline anisotropy in the opposite way compared to $\mathrm{CoFe}_{2} \mathrm{O}_{4}$ : compressive and tensile strain favor, respectively, out-of-plane and in-plane magnetization. We then discuss results of $a b$ initio calculations of the strain dependence of magnetocrystalline anisotropy. Finally, we provide simple arguments, based on second-order perturbation theory, which explain the opposite tendencies of the strain dependence of magnetocrystalline anisotropy in $\mathrm{CoCr}_{2} \mathrm{O}_{4}$ and $\mathrm{CoFe}_{2} \mathrm{O}_{4}$.

\section{EXPERIMENTAL AND COMPUTATIONAL DETAILS}

$\mathrm{CoCr}_{2} \mathrm{O}_{4}$ thin films were grown using pulsed-laser deposition (PLD). The RHEED (reflection high-energy electron diffraction) assisted PLD system is equipped with a Lambda Physik COMPex Pro 205-KrF laser with a wavelength of $248 \mathrm{~nm}$. The laser was run with a frequency of $0.5 \mathrm{~Hz}$ for $2 \mathrm{~h}$, using a laser fluence of $3 \mathrm{~J} / \mathrm{cm}^{2}$ and a target-substrate distance of $50 \mathrm{~mm}$, which resulted in $\mathrm{CoCr}_{2} \mathrm{O}_{4}$ films with thicknesses of about $40 \mathrm{~nm}$. The substrate was kept at $400-450{ }^{\circ} \mathrm{C}$ during growth. A ceramic $\mathrm{CoCr}_{2} \mathrm{O}_{4}$ pellet, sintered by solid-state reaction [33], was used as target. The growth took place in a 0.01-mbar oxygen plasma atmosphere, created by an Oxford Scientific mini-electron cyclotron resonance-plasma source in order to improve the oxidation of the films [34]. After deposition, the films were cooled down in 0.5 bar $_{2}$ atmosphere with a cooling rate of $5^{\circ} \mathrm{C}$ per minute. The structure of the thin films was determined by $\mathrm{X}$-ray diffraction (XRD), using an X'pert laboratory diffractometer. Magnetization was measured using a Quantum Design Magnetic Properties Measurement System 7 extra large superconducting quantum interference device magnetometer.

Thin films were deposited both on $\mathrm{MgO}$ and $\mathrm{MgAl}_{2} \mathrm{O}_{4}$ substrates. The substrates were postannealed at $650{ }^{\circ} \mathrm{C}$ overnight [35] in $1 \times 10^{-5}$-bar oxygen atmosphere. $\mathrm{MgAl}_{2} \mathrm{O}_{4}$ has the spinel crystal structure, like $\mathrm{CoCr}_{2} \mathrm{O}_{4}$, but with a smaller lattice parameter, corresponding to a lattice mismatch [36] of $3.1 \%$ that induces a large in-plane compressive strain in the $\mathrm{CoCr}_{2} \mathrm{O}_{4}$ films. $\mathrm{MgO}$ has the rock-salt structure with a lattice mismatch of $1.1 \%$, resulting in $\mathrm{CoCr}_{2} \mathrm{O}_{4}$ films under tensile strain.

$A b$ initio calculations were performed using the LSDA $+U$ approach, as implemented in the Vienna $A b$ Initio Simulation Package (VASP), with projector augmented wave

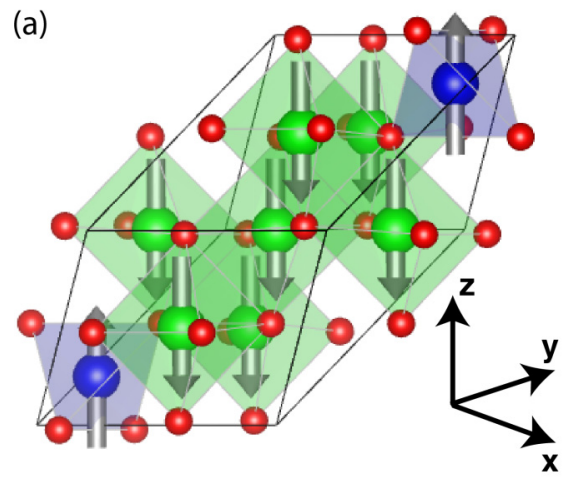

(b)

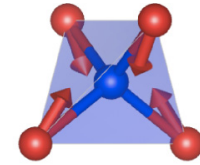

(c)

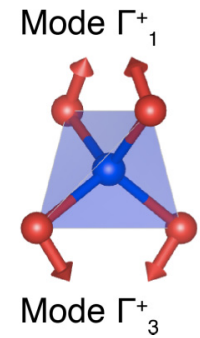

FIG. 1. (Color online) (a) Crystallographic structure of $\mathrm{CoCr}_{2} \mathrm{O}_{4}$. The primitive unit cell of the fcc lattice is shown, containing two formula units. Blue, green, and red spheres represent, respectively, $\mathrm{Co}^{2+}, \mathrm{Cr}^{3+}$, and $\mathrm{O}^{2-}$ ions. Gray arrows indicate the ferrimagnetic ordering considered in the calculations. Red arrows in panels (b) and (c) show the oxygen displacements for the two modes which appear on relaxing the structure under strain.

pseudopotentials. We use a $7 \times 7 \times 7$ Monkhorst $k$-point grid and consider the primitive unit cell of the underlying face-centered cubic (fcc) lattice, containing two formula units. The values of the effective on-site Coulomb interactions and Hund's couplings for $\mathrm{Co}^{2+}$ and $\mathrm{Cr}^{3+}$ were set to $\mathrm{U}_{\mathrm{Co}}=4 \mathrm{eV}$, $\mathrm{J}_{\mathrm{Co}}^{\mathrm{H}}=1 \mathrm{eV}$ and $\mathrm{U}_{\mathrm{Cr}}=3 \mathrm{eV}, \mathrm{J}_{\mathrm{Cr}}^{\mathrm{H}}=1 \mathrm{eV}$, respectively. As shown in Ref. [37], a realistic description of the magnetic properties of $\mathrm{CoCr}_{2} \mathrm{O}_{4}$ is obtained using these values. Moreover, for both transition-metal ions, the $3 s$ and $3 p$ states were treated as valence electrons. The lattice constants and atomic positions, both for the unstrained cubic unit cell and for various values of strain, were obtained by calculating the energy of relaxed structures at different values of the out-of-plane lattice constant and extracting the value corresponding to minimal energy. Relaxations were performed considering a collinear ferrimagnetic state in which the spins of the $\mathrm{Co}^{2+}$ sublattices are antiparallel to those of the $\mathrm{Cr}^{3+}$ sublattices [see Fig. 1(a)]. The normal spinel configuration considered in the calculations is consistent with the experimental evidence, from highresolution transmission electron microscopy experiments [38], supporting the normal-type spinel structure in the $\mathrm{CoCr}_{2} \mathrm{O}_{4}$ thin films under strain.

Calculations to obtain the size of the magnetocrystalline anisotropy were performed using noncollinear spin-polarized calculations, in which the direction of the spins of each magnetic sublattice was constrained and rotated, keeping the ferrimagnetic order described above.

\section{RESULTS AND DISCUSSION}

\section{A. Experimental results}

Despite the lattice mismatch between both substrates and $\mathrm{CoCr}_{2} \mathrm{O}_{4}$, high-quality epitaxial thin films have been grown under both tensile strain, on [001]-oriented $\mathrm{MgO}$ substrates, and under compressive strain, on [001]-oriented $\mathrm{MgAl}_{2} \mathrm{O}_{4}$ substrates. The possibility of applying high amounts of strain to spinel materials was also observed in $\mathrm{CoFe}_{2} \mathrm{O}_{4}$ by Gatel et al. [13]. The XRD characterization of the films shows 

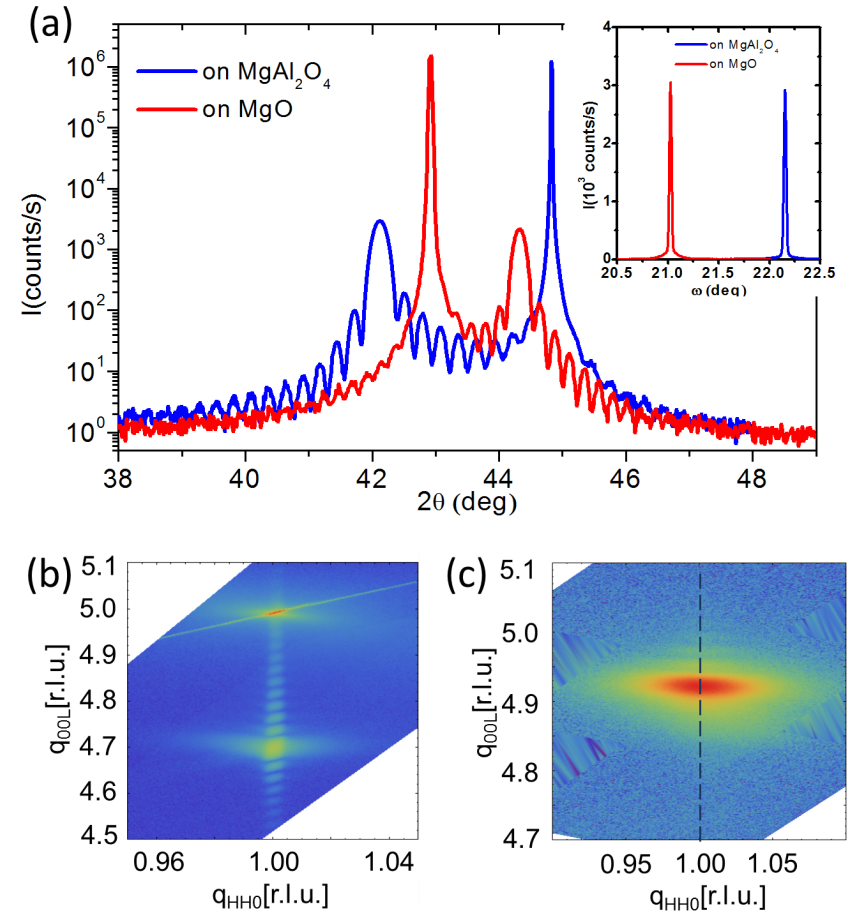

FIG. 2. (Color online) (a) Specular XRD pattern around the (004) $\mathrm{CoCr}_{2} \mathrm{O}_{4}$ reflections. The inset shows the rocking curves around the (004) reflections for both films. XRD Reciprocal space maps (RSMs) around the (115) reflections of the $\mathrm{CoCr}_{2} \mathrm{O}_{4}$ films grown on $\mathrm{MgAl}_{2} \mathrm{O}_{4}$ (b) and $\mathrm{MgO}$ (c) are shown. The axes in (b) and (c) are labeled in reciprocal-lattice units (r.l.u.) of the substrate. The vertical dashed line in (c) marks the position of the substrate peak (not shown).

a large number of thickness fringes around the film peaks, revealing a superb flatness of the film interfaces [Fig. 2(a)]. The excellent crystalline quality of the films is shown by the rocking curves of the film peak, displaying full width at half maximum (FWHM) below $0.03^{\circ}$ [inset of Fig. 2(a)]. The films on $\mathrm{MgAl}_{2} \mathrm{O}_{4}$ and $\mathrm{MgO}$ have out-of-plane lattice constants of 8.58 and $8.17 \AA$, respectively (bulk value is $c=8.33 \AA$ ).

The reciprocal space map around the (115) reflection of $\mathrm{CoCr}_{2} \mathrm{O}_{4}$ in Fig. 2(b) shows that the in-plane lattice constant of the films is identical to that of the $\mathrm{MgAl}_{2} \mathrm{O}_{4}$ substrate, as substrate and film peaks are observed at the same value of $\mathrm{q}_{H K 0}$. Thus, the films are coherently strained, despite the huge lattice mismatch between the $\mathrm{MgAl}_{2} \mathrm{O}_{4}$ and bulk $\mathrm{CoCr}_{2} \mathrm{O}_{4}$ of $3.1 \%$. Despite the relatively low intensity of this reflection, thickness fringes are also visible around the (115)-film peak.

According to our specular XRD measurements, $\mathrm{CoCr}_{2} \mathrm{O}_{4}$ films grown under tensile strain on $\mathrm{MgO}$ display a similar crystal quality to that of the films grown on $\mathrm{MgAl}_{2} \mathrm{O}_{4}$. The (115)-off-specular peak of $\mathrm{CoCr}_{2} \mathrm{O}_{4}$ in Fig. 2(c) shows that the film on $\mathrm{MgO}$ is also fully strained. However, the rocking curve of this peak (scan in the $\mathrm{q}_{H H 0}$ direction), has a FWHM of $1.2^{\circ}$. This is the result of the difference in structure between the rock-salt $\mathrm{MgO}$ substrate and the spinel $\mathrm{CoCr}_{2} \mathrm{O}_{4}$ film. It is known that the growth of spinel films on rock-salt structure materials can result in the formation of antiphase boundaries (APB), creating a less homogeneous lattice in the in-plane
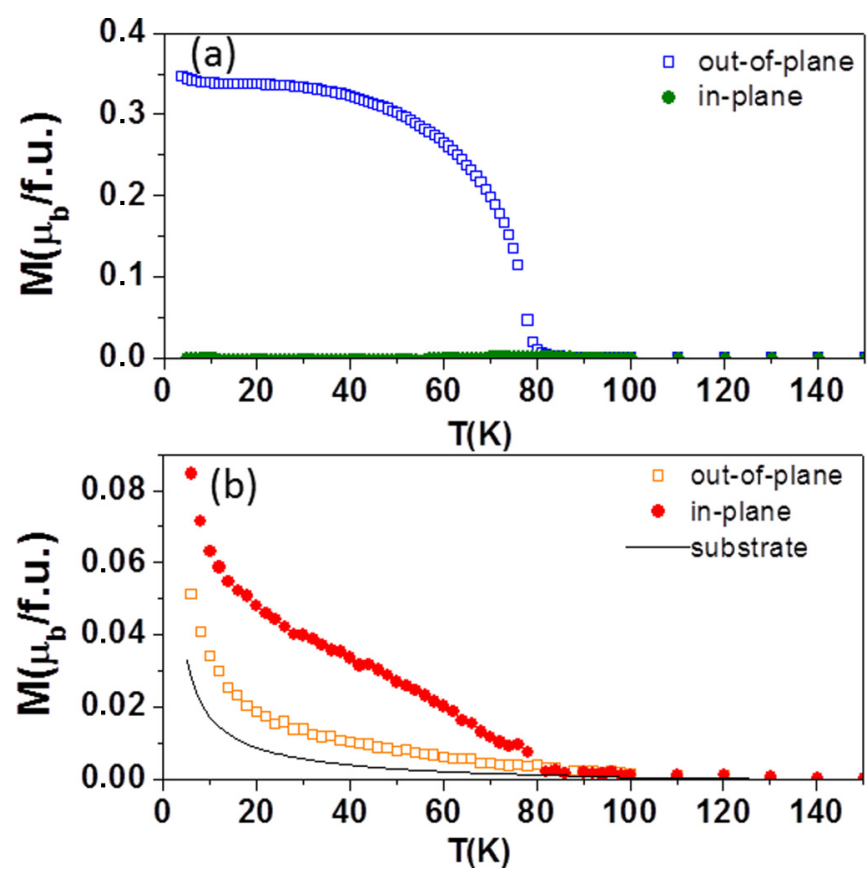

FIG. 3. (Color online) Magnetization of strained $\mathrm{CoCr}_{2} \mathrm{O}_{4}$ on $\mathrm{MgAl}_{2} \mathrm{O}_{4}$ (a) and $\mathrm{MgO}$ (b) measured both in plane and out of plane, in a field of $0.01 \mathrm{~T}$ after cooling in $1 \mathrm{~T}$. The magnetic response of a bare $\mathrm{MgO}$ substrate is added as a solid line [39].

directions. Antiphase boundaries have, indeed, been observed in our $\mathrm{CoCr}_{2} \mathrm{O}_{4}$ thin films using TEM. Their formation and effects will be reported elsewhere.

Magnetization of the thin films was measured in both in-plane and out-of-plane geometries. The magnetization of $\mathrm{CoCr}_{2} \mathrm{O}_{4}$ is highly anisotropic as shown in Fig. 3. Films on $\mathrm{MgAl}_{2} \mathrm{O}_{4}$, under compressive strain, in a field of $0.01 \mathrm{~T}$ applied perpendicular to the film, show the ferrimagnetic transition at $81 \mathrm{~K}$ (the bulk value being $T_{N 1} \sim 95 \mathrm{~K}$ ). Applying a magnetic field along the [100] in-plane direction induces only a paramagnetic tail, which originates partially from the substrate contribution, showing that the film has an easy out-of-plane axis. The films on $\mathrm{MgO}$, under tensile strain, show a ferrimagnetic transition at $\sim 80 \mathrm{~K}$ under a $0.01-\mathrm{T}$ field applied in the [100] in-plane direction, while no ferrimagnetic transition is observed when the field is applied in the out-ofplane direction. The magnetic anisotropy in $\mathrm{CoCr}_{2} \mathrm{O}_{4}$ films, thus, changes sign depending on the sign of the strain: the magnetic easy axis is out of plane in films under compressive strain, while it is in plane in films under tensile strain. As discussed above, the strain tuning of the magnetic anisotropy has also been observed in films of $\mathrm{CoFe}_{2} \mathrm{O}_{4}$ but in that case the effect is opposite [8].

It should be noted here that there is no evidence of the spiral transition in these magnetic measurements. In previously published results on $\mathrm{CoCr}_{2} \mathrm{O}_{4}$ crystals, this transition was also less pronounced than the ferrimagnetic one [26] and even sometimes almost undetectable in the magnetic measurements [23]. Because of this, evidence of a transition is often provided by other measurements such as specific heat or dielectric measurements. Dielectric measurements performed on different films grown on both substrates (data not shown) 
show slight changes in the slope around the spin spiral phase transition, indicating that the conical spin spiral may indeed survive in $\mathrm{CoCr}_{2} \mathrm{O}_{4}$ in thin-film form.

The magnitude of the magnetization is also dependent on the sign of the strain. The magnetization at $20 \mathrm{~K}$ of the $\mathrm{CoCr}_{2} \mathrm{O}_{4}$ films under compressive strain is $0.34 \mu_{B}$ per formula unit (f.u.), while it is only $0.03 \mu_{B} /$ f.u. under tensile strain. These values are, respectively, larger and smaller than the $0.08 \mu_{B} /$ f.u. found in single crystals [23]. Cation inversion is well known to increase or decrease magnetization in spinel structures as for instance in $\mathrm{NiFe}_{2} \mathrm{O}_{4}[21,40,41]$. However, $\mathrm{NiFe}_{2} \mathrm{O}_{4}$ and $\mathrm{CoFe}_{2} \mathrm{O}_{4}$ have a collinear ferrimagnetic structure, whereas in bulk $\mathrm{CoCr}_{2} \mathrm{O}_{4}$ the low-temperature magnetic structure is a ferrimagnetic conical spiral in which the low-temperature magnetization is not determined by cation inversion ( $\mathrm{Co}^{2+}$ and $\mathrm{Cr}^{3+}$ both have three unpaired spins) but by the conicities of the magnetic spirals of the various sublattices.

A ferrimagnetic conical spiral can also be present in thin-film samples at low temperatures. This, together with a possible dependence of the spiral conicities on the applied strain, might explain the change in the low-temperature magnetization. It is also worth noting that the gyromagnetic tensor is likely to depend weakly on strain, which might contribute to the discrepancy between the low-temperature magnetization of the two samples. Moreover, the magnetization of spinel structures on $\mathrm{MgO}$ can be smaller than in bulk as a result of antiphase boundaries $[9,10,42,43]$. As mentioned above, antiphase boundaries were observed in our $\mathrm{CoCr}_{2} \mathrm{O}_{4}$ films on $\mathrm{MgO}$, and could thus explain their lower magnetization values. However, since no anti-phase boundaries have been observed in our films on $\mathrm{MgAl}_{2} \mathrm{O}_{4}$, it is likely that the increased value of magnetization with respect to bulk $\mathrm{CoCr}_{2} \mathrm{O}_{4}$ in this case has other origins, such as changes in conicities.

The strain-dependent magnetic anisotropy is confirmed by the magnetic hysteresis measurements shown in Fig. 4. Films on $\mathrm{MgAl}_{2} \mathrm{O}_{4}$ [Fig. 4(a)] measured with the field along the outof-plane direction, show a square loop with coercive field of 2.2 $\mathrm{T}$ when the diamagnetic slope of the substrate contribution is subtracted, while in the in-plane direction no loop opens. This confirms that the [001] out-of-plane axis is an easier axis than the 100 in-plane axes. The hysteresis loop on $\mathrm{MgO}$ has an opening when the field is applied in the [100] in-plane direction while no loop opening is observed in the out-of-plane direction [Fig. 4(b)]. On MgO, the loop in the in-plane direction is not square, which could be a sign of the field not being applied along the easy axis. However, it is well known that films of $\mathrm{CoFe}_{2} \mathrm{O}_{4}$ with antiphase boundaries have such loop shapes $[18,42]$. In our $\mathrm{CoCr}_{2} \mathrm{O}_{4}$ films on $\mathrm{MgAl}_{2} \mathrm{O}_{4}$, we observe an extremely large coercive field (unusual for spinels), which most likely originates in the symmetry lowering induced by the strain. Extremely high coercive fields in spinel thin films have also been observed by Yanagihara et al. [44].

\section{B. Ab initio calculation of magnetic anisotropy}

To investigate the microscopic mechanisms leading to the strain dependence of the magnetic anisotropy shown in Fig. 3, we perform $a b$ initio calculations. We first calculate the crystal structure under strain by relaxing the out-of-plane lattice constant together with the atomic positions to their lowest-energy (a)

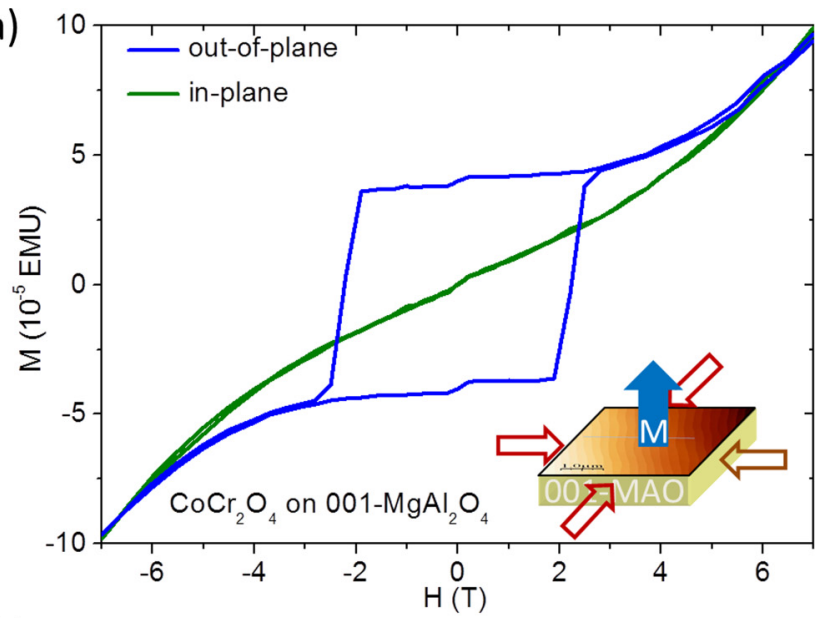

(b)

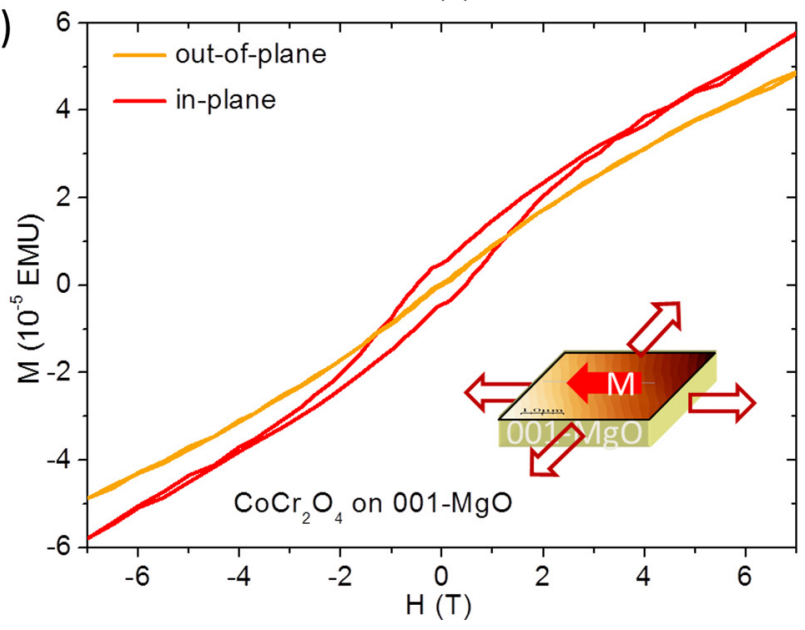

FIG. 4. (Color online) Magnetic hysteresis loops of $\mathrm{CoCr}_{2} \mathrm{O}_{4}$ on $\mathrm{MgAl}_{2} \mathrm{O}_{4}$, under compressive strain (a), and on $\mathrm{MgO}$, under tensile strain (b), at $20 \mathrm{~K}$, measured in the (100) in-plane and (001) out-ofplane directions.

value for a set of in-plane lattice constants. We then use the relaxed structures to calculate the magnetocrystalline anisotropy.

In addition to the change of the lattice constants, the structural relaxation of the strained unit cell induces two structural distortions involving oxygen displacements. These correspond to displacement modes of symmetry $\Gamma_{1}^{+}$and $\Gamma_{3}^{+}$. Mode $\Gamma_{1}^{+}$corresponds to a uniform compression (enlargement) of the oxygen tetrahedra coordinating $\mathrm{Co}^{2+}$ when compressive (tensile) strain is applied [see Fig. 1(b)]. Mode $\Gamma_{3}^{+}$, on the other hand, changes the local symmetry from that of a perfect tetrahedron to that of a tetragonally distorted one, as shown in Fig. 1(c). We note that the displacements of $\mathrm{O}^{2-}$ in mode $\Gamma_{3}^{+}$give rise to shifts in the crystal-field splitting similar to those corresponding to the change of the lattice constants when strain is applied. For example, mode $\Gamma_{3}^{+}$, when compressive (tensile) strain is applied, shrinks (enlarges) the tetrahedra surrounding $\mathrm{Co}^{2+}$ in the $x y$ plane and elongates (shrinks) them along the $z$ axis.

To estimate magnetocrystalline anisotropies we consider the anisotropic part of the magnetic Hamiltonian

$$
H_{A}=\sum_{i, v} \Delta_{v}^{(2)}\left(S_{i, v}^{z}\right)^{2}+\sum_{i, v} \Delta_{v}^{(4)}\left[\left(S_{i, \nu}^{x}\right)^{4}+\left(S_{i, \nu}^{y}\right)^{4}+\left(S_{i, \nu}^{z}\right)^{4}\right] \text {, }
$$



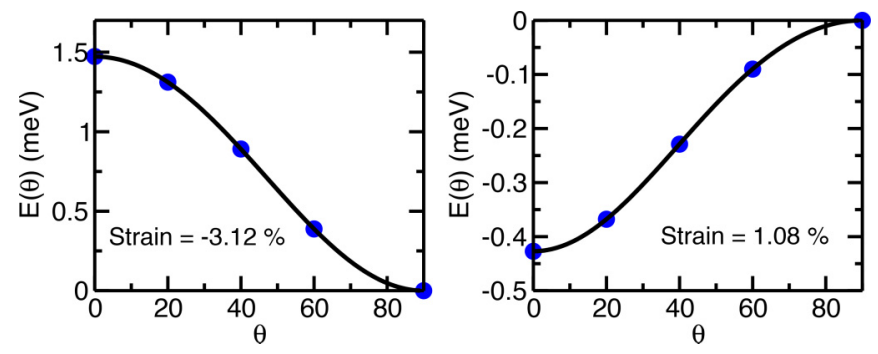

FIG. 5. (Color online) The left and right panels show, respectively, the calculated angle $(\theta)$ dependence of the energy (with the energy at $\theta=90^{\circ}$ subtracted) for $-3.12 \%$ (compressive) and $1.08 \%$ (tensile) strain. $\theta=0$ corresponds to the magnetic moment along the $x$ axis (in-plane), while $\theta=90^{\circ}$ corresponds to the magnetic moment along $z$ (out of plane). The two values of the strain are approximately equal to the experimental ones.

where $i$ and $v=1, \ldots, 6$ label, respectively, the unit cell and the magnetic sublattices in the unit cell; $\Delta_{v}^{(2)}$ and $\Delta_{v}^{(4)}$ are, respectively, the strength of quadratic and quartic anisotropy of spins at sublattice $v$; and $S^{\alpha}$, with $\alpha=x, y, z$, are the spin components along the pseudocubic crystallographic axes. Here we denote with $z$ the direction perpendicular to the film. On the one hand, for the bulk case, the first term on the right-hand side of Eq. (1) has to vanish due to the cubic symmetry. On the other hand, while the fourth-order term is always allowed, relatively small tetragonal distortions are expected to make the second-order term dominating.

We map ab initio energies to the Hamiltonian in Eq. (1) using the following procedure (see, e.g., Ref. [45]). We perform a set of $a b$ initio calculations where the direction of all the spins is rotated uniformly (i.e., keeping the magnetic ordering of Fig. 1) from the in-plane direction $(x)$ to the out-of-plane direction $(z)$ by an angle $\theta$. For these magnetic configurations, Eq. (1) yields a $\theta$ dependence of the $a b$ initio energy per unit cell given by

$$
\epsilon(\theta)=\alpha \cos (\theta)^{2}+\beta\left[\cos (\theta)^{4}+\sin (\theta)^{4}\right]+\gamma .
$$

Here, $\gamma$ is a constant energy shift which includes all contributions unrelated to magnetocrystalline anisotropy, while $\alpha$ and $\beta$ include, respectively, the quadratic and quartic contributions to spin anisotropy of all six magnetic sublattices in the unit cell.

The two panels in Fig. 5 show the numerical results (dots) and the fits (curves) obtained using Eq. (2) for $\epsilon(\theta)$ for strains close to the experimental values of $-3.1 \%$ (compressive) and $1.1 \%$ (tensile). We note that $\epsilon(\theta)$ fits well the $a b$ initio energies, indicating that the model in Eq. (1) describes well the magnetocrystalline anisotropies in this compound. Moreover, we find that, in agreement with experimental results, a tensile value of the strain favors a direction of the spins in the film plane (energy minimum at $\theta=0^{\circ}$ ), while compressive strain favors a direction perpendicular to the film plane (energy minimum at $\theta=90^{\circ}$ ).

For the case of a collinear ferrimagnetic state, the magnetocrystalline anisotropy part of the microscopic magnetic Hamiltonian, Eq. (1), can be easily related to the direction of magnetization, i.e., magnetization is favored along the easy axis anisotropy direction. However, for a ferrimagnetic conical

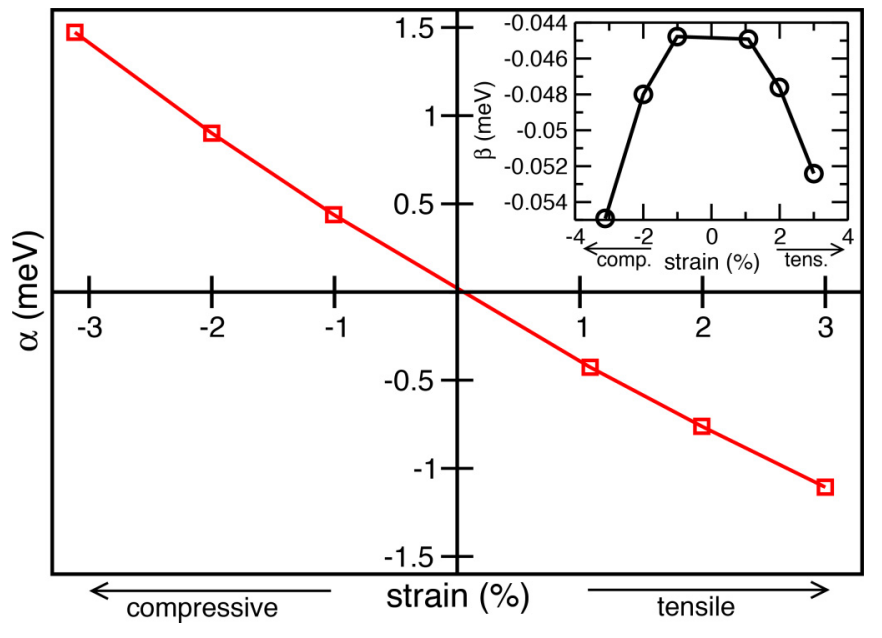

FIG. 6. (Color online) Calculated strain dependence of the constant $\alpha$, as described in the text. The inset shows the strain dependence of the constant $\beta$ related to the quartic anisotropy.

spiral state, such a straightforward relationship does not always hold. In this state, the magnetic ordering of the various sublattices consists of a collinear and a spiral component and the collinear component, which gives rise to the net magnetization, is perpendicular to the plane containing the spiral one. Therefore, whether the collinear or the spiral part of the magnetic ordering is along the easy axis direction might be related to the conicity of the spin ordering, i.e., the relative size of the collinear component with respect to the spiral one. Nonetheless, we note that the transition temperatures in Fig. 3 are comparable with the transition temperature of the collinear ferrimagnetic state in the bulk $\left(T_{N 1} \sim 95 \mathrm{~K}\right)$. It is thus reasonable to assume that, at least at temperatures below but close to the transition, the magnetic ordering is collinear also for the films and, thus, the magnetization is parallel to the microscopic easy direction given by Eq. (1).

To generalize the dependence of the magnetocrystalline anisotropy on strain, we extract the values of the constants $\alpha$ and $\beta$ as the strain is tuned. These dependences are shown in Fig. 6 . We note that the strength of the quadratic part $(\alpha)$ of the magnetocrystalline anisotropy decreases linearly (to first approximation) as the strain is tuned from compressive to tensile and changes sign for the bulk structure. In contrast, the strength of the quartic term $(\beta)$ shows a much weaker dependence on strain.

It is reasonable to assume that the strongest contribution to the magnetocrystalline anisotropy originates from the $\mathrm{Co}$ sublattice due to the $d^{7}$ electronic configuration of $\mathrm{Co}^{2+}$ and the larger value of spin-orbit coupling (which increases with the atomic number) compared to $\mathrm{Cr}^{3+}$. To verify this assumption, we estimate the separate contributions of the $\mathrm{Co}^{2+}$ and $\mathrm{Cr}^{3+}$ sublattices by performing the following calculations. We constrain the spins of the $\mathrm{Cr}$ sublattices to be ferromagnetically aligned along the $y$ direction and the spins of the Co sublattice to be ferromagnetically aligned along a direction in the $x z$ plane forming an angle $\theta_{\mathrm{Co}}$ with the $x$ axes. As $\theta_{\text {Co }}$ is changed, the exchange energy within the $\mathrm{Co}(\mathrm{Cr})$ sublattices cannot vary, as all the spins within these sublattices are kept parallel. Moreover, the exchange 


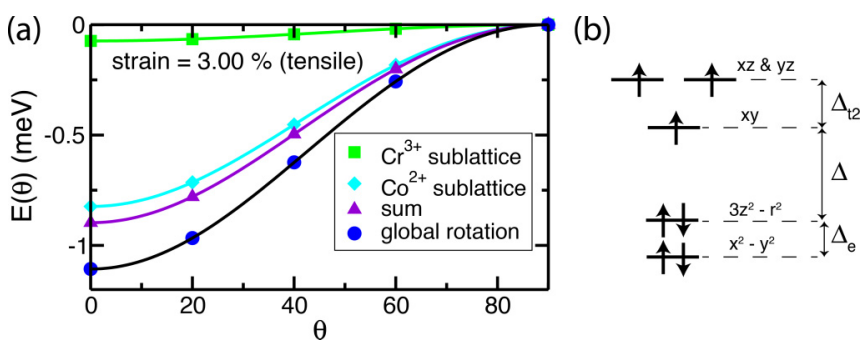

FIG. 7. (Color online) (a) Calculated energy dependence on the angle $\theta$ when the contributions of the $\mathrm{Cr}^{3+}$ (green squares) and $\mathrm{Co}^{2+}$ (light blue diamonds) are separated as described in the text for $3 \%$ tensile strain. The sum of the two contributions and the energy dependence obtained by a global rotation of all the spins are shown by (violet) triangles and (blue) dots, respectively. Curves represent the fits obtained as described in the text. (b) Crystal-field splitting of $\mathrm{a} \mathrm{Co}^{2+}$ ion in tetrahedral coordination under compressive strain.

energy between the $\mathrm{Co}$ and $\mathrm{Cr}$ sublattices is always zero, as their spins are kept perpendicular to each other. Therefore, the main contribution to the spin part of the Hamiltonian should originate from Eq. (1), which, for such a spin configuration, has the energy dependence

$$
\epsilon\left(\theta_{\mathrm{Co}}\right)=\alpha_{\mathrm{Co}} \cos \left(\theta_{\mathrm{Co}}\right)^{2}+\beta_{\mathrm{Co}}\left[\cos \left(\theta_{\mathrm{Co}}\right)^{4}+\sin \left(\theta_{\mathrm{Co}}\right)^{4}\right] .
$$

Here, $\alpha_{\mathrm{Co}}$ and $\beta_{\mathrm{Co}}$ contain the contribution to the anisotropy of the $\mathrm{Co}^{2+}$ sublattice and a constant energy shift is omitted. Similar calculations can be performed to obtain the anisotropy contributions of only the $\mathrm{Cr}^{3+}$ sublattice, $\alpha_{\mathrm{Cr}}$ and $\beta_{\mathrm{Cr}}$.

Figure 7(a) shows the comparison of the contributions of the $\mathrm{Co}$ and $\mathrm{Cr}$ sublattices to the magnetocrystalline anisotropy for the case of $3 \%$ tensile strain. In agreement with our initial assumption, the magnetocrystalline anisotropy is dominated by the contribution of Co ions. Interestingly, we note that the sum of the contributions of the two sublattices does not give exactly the value obtained for the total magnetocrystalline anisotropy. We further investigated the origin of such difference and excluded possible sizable contributions from terms such as Dzyaloshinskii-Moriya interaction or anisotropies of the exchange interactions. A possible source of this discrepancy might arise from a nonlocal contribution to the magnetocrystalline anisotropy, e.g., a contribution related to hopping between $\mathrm{Co}^{2+}$ and $\mathrm{Cr}^{3+}$ which might depend on the relative orientation of the spins of the two sublattices.

\section{Second-order perturbation theory for the magnetocrystalline anisotropy}

After establishing that the dominant contribution to magnetocrystalline anisotropy originates from the $\mathrm{Co}^{2+}$ sublattices, we note that the strain dependence of the anisotropy can be understood using a simple picture of isolated $\mathrm{Co}^{2+}$ ions coordinated by distorted oxygen tetrahedra. In this case the $\mathrm{Co}^{2+}$ contribution to the first term on the right-hand side of Eq. (1) can be obtained by second-order perturbation theory in the spin-orbit coupling constant $\lambda[46,47]$. This gives the expression

$$
H_{A}=\Delta_{\mathrm{Co}}^{(2)}\left(S^{z}\right)^{2}=-\lambda^{2}\left(\Lambda^{z z}-\Lambda^{x x}\right)\left(S^{z}\right)^{2},
$$

where we treat the Co spin as a classical vector and $\Lambda^{i i}=$ $\sum_{k} \frac{\left|\left\langle 0\left|L_{i}\right| k\right\rangle\right|^{2}}{\varepsilon_{k}-\varepsilon_{0}}$. Here, $L_{i}$ is the $i$ th component of the orbital momentum operator, and $|k\rangle$ and $|0\rangle$ denote, respectively, the $k$ th excited state and the ground state. To obtain $H_{A}$, we use the tetragonal symmetry, i.e., $\Lambda^{x x}=\Lambda^{y y}$.

We first note that for the case of a ("normal") cubic spinel the symmetry of the ions sitting in the $\mathbf{T}$ sites is $\mathbf{T}_{d}$. Considering the joint effect of the change of the lattice constants and the mode $\Gamma_{3}^{+}$, one obtains (for the case of compressive strain) the splitting of the $d$ levels as sketched in Fig. 7(b). Here, we consider the $d^{7}$ electronic configuration and we assume that $\Delta>\Delta_{t 2}>\Delta_{e}>0$, as the lobes of $t_{2}$ states are pointing more directly toward the ligands. We also note that, in principle, crystal-field splittings can be estimated from the $a b$ initio calculations using Wannier functions. However, it was shown (see, e.g., Ref. [48]) that this method requires a careful analysis and the so-obtained splittings do not necessarily correspond to a simple point charge model of isolated ions within a crystal field. By taking into account only the electronic excitations with lowest energy, i.e., only one-electron excitations not violating Hund's rule, we obtain

$$
\Delta_{\mathrm{Co}-\mathrm{T}}^{(2)}=\frac{\lambda^{2}\left[-4 \Delta_{t 2}\left(\Delta+\Delta_{t 2}\right)+3 \Delta_{e}\left(\Delta+\Delta_{e}\right)\right]}{\left(\Delta+\Delta_{t 2}\right)\left(\Delta+\Delta_{e}\right)\left(\Delta+\Delta_{e}+\Delta_{t 2}\right)},
$$

where the subscript $\mathrm{T}$ indicates that the equation holds for $\mathrm{Co}^{2+}$ in tetrahedral coordination. This expression, for the case of compressive strain, leads to an out-of-plane easy axis anisotropy $\left(\Delta_{\mathrm{Co}}^{(2)}\right.$ is negative as $\Delta_{t 2}>\Delta_{e}$, which favors spin orientation along $z$ ), in qualitative agreement both with experiments and $a b$ initio calculations. Moreover, we note that Eq. (5) holds also for the case of tensile strain, where $\Delta_{t 2}$ and $\Delta_{e}$ are expected to be negative with $\Delta_{t 2}<\Delta_{e}<0$. This gives rise to an easy-plane value of the quadratic anisotropy (positive $\Delta_{\mathrm{Co}}^{(2)}$, which favors spin orientation perpendicular to $z$ ).

Furthermore, we speculate that a similar argument can be used to explain the sign of the magnetostriction in the inverse spinel $\mathrm{CoFe}_{2} \mathrm{O}_{4}$. Assuming that also in $\mathrm{CoFe}_{2} \mathrm{O}_{4}$ the largest contribution to the magnetocrystalline anisotropy originates from the $\mathrm{Co}^{2+}$ ions, we use similar considerations to those used for the tetrahedrally coordinated $\mathrm{Co}^{2+}$ to obtain an expression for the anisotropy for the case of octahedral coordination. It is important to note that in a cubic spinel (both normal and inverse) the octahedral sites do not possess symmetry $\mathbf{O}_{h}$ but are trigonally distorted. Such distortion was previously shown to play an important role in the surprisingly large quartic magnetic anisotropy found in cubic $\mathrm{Co}_{x} \mathrm{Fe}_{3-x} \mathrm{O}_{4}$ $[19,20]$. Nonetheless, as we are interested in the emergence of second-order anisotropy with a strain-induced tetragonal distortion, we consider the case of a perfect $\mathbf{O}_{h}$ symmetry for the unstrained case. Moreover, we restrict ourselves to the case of compressive strain where, similarly to the case of $\mathrm{CoCr}_{2} \mathrm{O}_{4}$, nondegenerate perturbation theory can be applied [47]. For compressive strain, we obtain

$$
\Delta_{\mathrm{Co}-\mathrm{O}}^{(2)}=\frac{\lambda^{2}\left[\Delta_{t 2 g}\left(4 \Delta_{e g}+5 \Delta_{t 2 g}\right)+\Delta\left(\Delta+\Delta_{e g}+6 \Delta_{t 2 g}\right)\right]}{\Delta_{t 2 g}\left(\Delta+\Delta_{t 2 g}\right)\left(\Delta+\Delta_{e g}+\Delta_{t 2 g}\right)},
$$


where the subscript $\mathrm{O}$ indicates octahedral coordination, $\Delta_{t 2 g}=\varepsilon_{x y}-\varepsilon_{x z / y z}, \Delta_{e g}=\varepsilon_{x^{2}-y^{2}}-\varepsilon_{z^{2}}, \Delta=\varepsilon_{z^{2}}-\varepsilon_{x y}$, and $\varepsilon_{i}$ is the on-site energy of the $i$ th $d$ orbital. For compressive strain of the octahedron, it is reasonable to assume that $\Delta_{e g}>$ $0, \Delta_{t 2 g}>0$ and $\Delta>0$, which implies that the anisotropy, Eq. (6), favors in-plane orientation of the spins $\left(\Delta_{\mathrm{Co}-\mathrm{O}}^{(2)}>0\right)$. We stress that in contrast to Eq. (5) the expression in Eq. (6) does not hold for tensile strain as the treatment of this case would require the use of degenerate perturbation theory.

\section{SUMMARY}

We report the growth of fully strained (001) $\mathrm{CoCr}_{2} \mathrm{O}_{4}$ films on $\mathrm{MgAl}_{2} \mathrm{O}_{4}$ and $\mathrm{MgO}$ substrates. Magnetometry experiments reveal that compressively strained $\mathrm{CoCr}_{2} \mathrm{O}_{4}$ thin films exhibit a perpendicular magnetization, while films under tensile strain exhibit an in-plane magnetization. The mapping of LSDA $+U$ calculations to the anisotropic part of an effective spin model, Eq. (1), shows that (001) compressive strain favors a spin easy axis along the [001] direction while tensile strain favors spin orientation in the film plane. The quadratic anisotropy term changes smoothly as strain is tuned and vanishes in the cubic case. Moreover, these calculations confirm that the main contribution to magnetocrystalline anisotropy is given by the $\mathrm{Co}^{2+}$ sublattice. The direction of magnetization is parallel to the easy direction of the microscopic magnetic model for the collinear ferrimagnetic state and for ferrimagnetic conical states with large enough conicity. We note that, for both the samples, the ferrimagnetic state appears at temperature close to $\mathrm{T}_{N 1}$ below which bulk $\mathrm{CoCr}_{2} \mathrm{O}_{4}$ is collinear for a large temperature regime $(26 \lesssim \mathrm{T} \lesssim 95 \mathrm{~K})$. Therefore, it is reasonable to assume that the magnetization is parallel to the easy direction of model Eq. (1). Under this assumption the results obtained with $\mathrm{LSDA}+U$ are in agreement with experimental observations.

Furthermore, to explain the behavior of the quadratic part of the magnetocrystalline anisotropy with strain in $\mathrm{CoCr}_{2} \mathrm{O}_{4}$, we give a simple argument based on a perturbative treatment of spin-orbit coupling when the crystal-field splitting is modified by strain. Finally, we use these arguments to explain why the strain dependence of anisotropy is opposite in the inverse spinel $\mathrm{CoFe}_{2} \mathrm{O}_{4}$. The same sign of the strain-induced anisotropy as in $\mathrm{CoFe}_{2} \mathrm{O}_{4}$ has been reported in $\mathrm{CoO}$ films [49] (also containing $\mathrm{Co}^{2+}$ in octahedral coordination), indicating that the simple argument given in our discussion might not be only specific for spinel structures but can, in principle, be extended to more general cases.
[1] S. Mangin, D. Ravelosona, J. A. Katine, M. J. Carey, B. D. Terris, and E. E. Fullerton, Nat. Mater. 5, 210 (2006).

[2] S. Ikeda, K. Miura, H. Yamamoto, K. Mizunuma, H. D. Gan, M. Endo, S. Kanai, J. Hayakawa, F. Matsukura, and H. Ohno, Nat. Mater. 9, 721 (2010).

[3] K. Yakushiji, T. Saruya, H. Kubota, A. Fukushima, T. Nagahama, S. Yuasa, and K. Ando, Appl. Phys. Lett. 97, 232508 (2010).

[4] S. Fukami, T. Suzuki, Y. Nakatani, N. Ishiwata, M. Yamanouchi, S. Ikeda, N. Kasai, and H. Ohno, Appl. Phys. Lett. 98, 082504 (2011).

[5] H. Meng and J.-P. Wang, Appl. Phys. Lett. 88, 172506 (2006).

[6] N. A. Spaldin, Magnetic Materials (Cambridge University Press, Cambridge, England, 2003).

[7] P. C. Dorsey, P. Lubitz, D. B. Chrisey, and J. S. Horwitz, J. Appl. Phys. 79, 6338 (1996).

[8] Y. Suzuki, G. Hu, R. B. van Dover, and R. J. Cava, J. Magn. Magn. Mater. 191, 1 (1999).

[9] S. A. Chambers, R. F. C. Farrow, S. Maat, M. F. Toney, L. Folks, J. G. Catalano, T. P. Trainor, and G. E. Brown, J. Magn. Magn. Mater. 246, 124 (2002).

[10] A. Lisfi, C.-M. Williams, L.-T. Nguyen, J.-C. Lodder, A. Coleman, H. Corcoran, A. Johnson, P. Chang, A. Kumar, and W. Morgan, Phys. Rev. B 76, 054405 (2007).

[11] G. Hu, J.-H. Choi, C.-B. Eom, V.-G. Harris, and Y. Suzuki, Phys. Rev. B 62, R779 (2000).

[12] R. M. Bozorth, E. F. Tilden, and A. J. Williams, Phys. Rev. 99, 1788 (1955).

[13] C. Gatel, B. Warot-Fonrose, S. Matzen, and J.-B. Moussy, Appl. Phys. Lett. 103, 092405 (2013).

[14] D. Fritsch and C. Ederer, Phys. Rev. B 82, 104117 (2010).

[15] D. Fritsch and C. Ederer, Phys. Rev. B 86, 014406 (2012).
[16] Here $\mathbf{T}$ and $\mathbf{O}$ do not refer to the Schoenflies notation of the point group but label the type of coordination, respectively, tetrahedral and octahedral.

[17] M. Kriegisch, W. Ren, R. Sato-Turtelli, H. Müller, R. Grössinger, and Z. Zhang, J. Appl. Phys. 111, $07 E 308$ (2012).

[18] S. Matzen, J.-B. Moussy, R. Mattana, F. Petroff, C. Gatel, B. Warot-Fonrose, J. C. Cezar, A. Barbier, M.-A. Arrio, and P. Sainctavit, Appl. Phys. Lett. 99, 052514 (2011).

[19] J. C. Slonczewski, Phys. Rev. 110, 1341 (1958).

[20] M. Tachiki, Prog. Theor. Phys. 23, 1055 (1960).

[21] Z. Szotek, W.-M. Temmerman, D. Ködderitzsch, A. Svane, L. Petit, and H. Winter, Phys. Rev. B 74, 174431 (2006).

[22] D. Fritsch and C. Ederer, Appl. Phys. Lett. 99, 081916 (2011).

[23] Y. Yamasaki, S. Miyasaka, Y. Kaneko, J. P. He, T. Arima, and Y. Tokura, Phys. Rev. Lett. 96, 207204 (2006).

[24] G. Lawes, B. Melot, K. Page, C. Ederer, M. A. Hayward, T. Proffen, and R. Seshadri, Phys. Rev. B 74, 024413 (2006).

[25] K. Tomiyasu, J. Fukunaga, and H. Suzuki, Phys. Rev. B 70, 214434 (2004).

[26] Y.-J. Choi, J. Okamoto, D.-J. Huang, K.-S. Chao, H.-J. Lin, C.-T. Chen, M. van Veenendaal, T.-A. Kaplan, and S.-W. Cheong, Phys. Rev. Lett. 102, 067601 (2009).

[27] K. Dwight, J. Appl. Phys. 40, 1156 (1969).

[28] U. Lüders, F. Sánchez, and J. Fontcuberta, Mater. Sci. Eng. B 109, 200 (2004).

[29] Y. Suzuki, R. B. Van Dover, E. M. Gyorgy, J. M. Phillips, V. Korenivski, D. J. Werder, C. H. Chen, R. J. Cava, J. J. Krajewski, W. F. Peck, and K. B. Do, Appl. Phys. Lett. 68, 714 (1996).

[30] G. Hu and Y. Suzuki, Phys. Rev. Lett. 89, 276601 (2002).

[31] X. Liu, M. Kareev, Y. Cao, J. Liu, S. Middey, D. Meyers, J. W. Freeland, and J. Chakhalian, Appl. Phys. Lett. 105, 042401 (2014). 
[32] A. Navrotsky and O. J. Kleppa, J. Inorg. Nucl. Chem. 29, 2701 (1967).

[33] N. Mufti, A. A. Nugroho, G. R. Blake, and T. T. M. Palstra, J. Phys.: Condens. Matter 22, 075902 (2010).

[34] J. X. Ma, D. Mazumdar, G. Kim, H. Sato, N. Z. Bao, and A. Gupta, J. Appl. Phys. 108, 063917 (2010).

[35] W. Eerenstein, Spin-dependent transport across antiphase boundaries in magnetite films, Ph.D. thesis, University of Groningen, 2003.

[36] Here we consider as lattice mismatch $\left|a_{s}-a_{\mathrm{CCO}}\right| / a_{\mathrm{CCO}}$ where $a_{\mathrm{CCO}}$ is the bulk lattice constant of $\mathrm{CoCr}_{2} \mathrm{O}_{4}$ and $a_{s}$ is the lattice constant of the substrate in the direction relevant for the strain.

[37] C. Ederer and M. Komelj, Phys. Rev. B 76, 064409 (2007).

[38] R. Guzmán, J. A. Heuver, S. Matzen, B. Noheda, and C. Magén (unpublished).

[39] The substrate is measured before annealing, with the $\mathrm{H}$ field parallel to the in-plane direction. This particular substrate is not exactly the same as that on which the film in Fig. 3 was grown and, thus, the paramagnetic impurity tail may differ. Annealing of the substrate prior to deposition may also alter the impurity content.

[40] U. Lüders, M. Bibes, J. F. Bobo, M. Cantoni, R. Bertacco, and J. Fontcuberta, Phys. Rev. B 71, 134419 (2005).
[41] F. Rigato, S. Estradé, J. Arbiol, F. Peiró, U. Lüders, X. Martí, F. Sánchez, and J. Fontcuberta, Mater. Sci. Eng. B 144, 43 (2007).

[42] D. T. Margulies, F. T. Parker, M. L. Rudee, F. E. Spada, J. N. Chapman, P. R. Aitchison, and A. E. Berkowitz, Phys. Rev. Lett. 79, 5162 (1997).

[43] S. Venzke, R. B. Van Dover, J. M. Phillips, E. M. Gyory, T. Siegrist, C. H. Chen, D. Werder, R. M. Fleming, R. J. Felder, E. Coleman, and R. Opila, J. Mater. Res. 11, 1187 (1996).

[44] H. Yanagihara, K. Uwabo, M. Minagawa, E. Kita, and N. Hirota, J. Appl. Phys. 109, 07C122 (2011).

[45] C. Weingart, N. Spaldin, and E. Bousquet, Phys. Rev. B 86, 094413 (2012).

[46] P. Fazekas, Lecture Notes on Electron Correlation and Magnetism (World Scientific, Singapore, 1999).

[47] D. Dai, H. Xiang, and M.-H. Whangbo, J. Comput. Chem. 29, 2187 (2008).

[48] A. Scaramucci, J. Ammann, N. A. Spaldin, and C. Ederer, J. Phys.: Condens. Matter 27, 175503 (2015).

[49] S.-I. Csiszar, M.-W. Haverkort, Z. Hu, A. Tanaka, H.-H. Hsieh, H.-J. Lin, C.-T. Chen, T. Hibma, and L.-H. Tjeng, Phys. Rev. Lett. 95, 187205 (2005). 